\title{
FINITE ELEMENT IMPLEMENTATION OF TWO-EQUATION AND ALGEBRAIC STRESS TURBULENCE MODELS FOR STEADY INCOMPRESSIBLE FLOWS
}

\author{
RAMON CODINA* AND ORLANDO SOTO \\ International Center for Numerical Methods in Engineering, Universitat Politècnica de Catalunya, Gran Capità s/n, \\ Edifici C1, 08034 Barcelona, Spain
}

\begin{abstract}
SUMMARY
The main purpose of this paper is to describe a finite element formulation for solving the equations for $k$ and $\varepsilon$ of the classical $k-\varepsilon$ turbulence model, or any other two-equation model. The finite element discretization is based on the SUPG method together with a discontinuity capturing technique to deal with sharp internal and boundary layers. The iterative strategy consists of several nested loops, the outermost being the linearization of the Navier-Stokes equations. The basic $k-\varepsilon$ model is used for the implementation of an algebraic stress model that is able to account for the effects of rotation. Some numerical examples are presented in order to show the performance of the proposed scheme for simulating directly steady flows, without the need of reaching the steady state through a transient evolution. Copyright (C) 1999 John Wiley \& Sons, Ltd.
\end{abstract}

KEY WORDS: turbulence models; nested schemes; discontinuity-capturing

\section{INTRODUCTION}

The main objective of this paper is to present a finite element implementation of the $k-\varepsilon$ turbulence model for steady flows [1-3]. Both a description of the approximation technique and of the iterative strategy are presented to deal with the non-linearity of the problem.

The iterative strategy is based on several facts related to the structure of the $k-\varepsilon$ equations. First, the equations for $k$ and $\varepsilon$ are coupled iteratively; meaning that they are solved one after the other and then iterated to converge to the coupled solution. The equations to be solved are of non-linear convection-diffusion-reaction-type. Since the diffusion coefficient depends on $k$ and $\varepsilon$, it is kept frozen until converging for the rest on non-linearities and coupling. This is intended to avoid the possibility of having negative values of diffusion during the iterative process, which may lead to divergence of the scheme.

Another feature of the iterative strategy is related to the production terms. These may appear as reaction terms for the variable being solved $(k$ or $\varepsilon)$ with the sign of the coefficient multiplying this variable negative (if it is put in the left-hand-side of the equation). An overestimation of the value of this coefficient in the iterative process could lead to a

* Correspondence to: ETS d'Enginyers de Camins, CiP, Universitat Politècnica de Catalunya, Gran Capità s/n, Edifici C1, 08034 Barcelona, Spain.

CCC 0271-2091/99/110309-25\$17.50

Received September 1997

Copyright (C) 1999 John Wiley \& Sons, Ltd.

Revised April 1998 
deterioration of the stability of the equation. For this reason, the reactive-like terms that may have the 'wrong' sign from the stability point of view are kept constant. This leads to an outer loop, the convergence of which is checked in terms of the mixing length $L$ associated with the $k-\varepsilon$ model.

With the previous considerations in mind, the authors have designed an iterative scheme consisting of three nested loops, which, for each one, it is often useful to perform a relaxation of the variable that has just been computed.

Once the linearization of the equations to be solved has been done, their approximation is based on a finite element discretization in space using the SUPG method. Also, since sharp boundary and internal layers may appear for both $k$ and $\varepsilon$ due to the convection of these quantities and the production terms, a discontinuity capturing technique is used that is based on the introduction of a non-linear diffusion proportional to the element residual [4,5], thus keeping the numerical consistency of the original formulation. The need for such a technique comes from the fact that it is very important to avoid local oscillations of the turbulence parameters, since this may lead to divergence of the iterative scheme. It must taken into account that these turbulence parameters must be positive, and local oscillations may yield negative values.

The authors have checked the performance of the algorithm presented in this paper in several numerical tests. The proposed main advantage of the this approach with respect to existing methods is the possibility of solving steady flow problems without the need of reaching the steady state through a transient evolution. Also, the method turns out to be very robust, in the sense that it is applicable without the need of specifying special velocity boundary conditions and works using different types of finite elements (linear and quadratic). The formulation proposed herein compares favourably with other methods, such as the classical

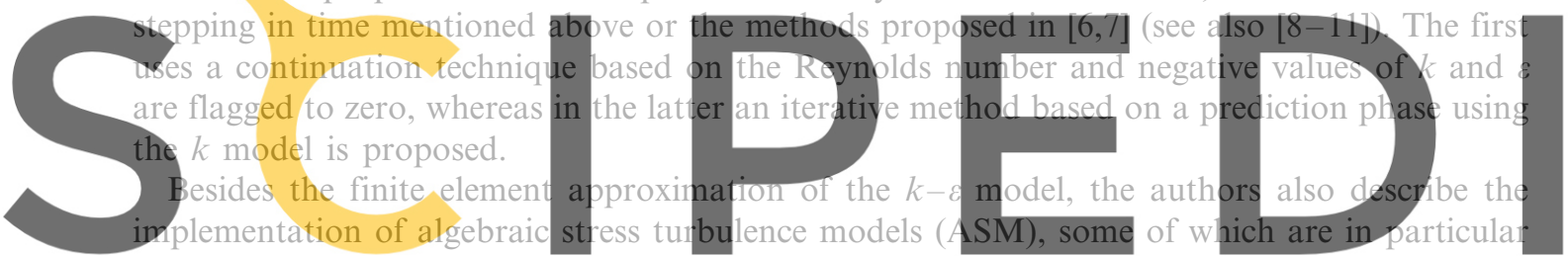

able to take into account the effects of the Coriolis force in rotating fluids [12-14]. These models give a closed expression for the Reynolds stresses in terms of $k$ and $\varepsilon$. Sometimes they

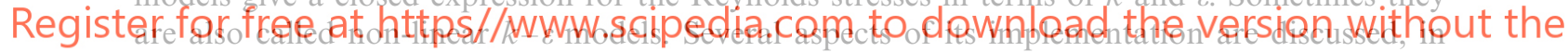

particular, the preconditioning based on the $k-\varepsilon$ model.

The paper has been organized as follows. In the next section, the problem to be solved is described. The iterative strategy is presented in Section 3, whereas the finite element approximation is presented in Section 4. Once the numerical model is described, some numerical examples are presented in Section 5 and some conclusions are drawn in Section 6.

\section{PROBLEM DESCRIPTION}

\subsection{Equations of motion}

The steady state Navier-Stokes equations for a turbulent incompressible fluid moving in a domain $\Omega$ can be written as

$$
(\boldsymbol{u} \cdot \nabla) \boldsymbol{u}-\nabla \cdot[2 v \boldsymbol{S}(\boldsymbol{u})+\tau]+\nabla p=\boldsymbol{f}
$$




$$
\nabla \cdot \boldsymbol{u}=0
$$

where $\boldsymbol{u}$ is the mean velocity field, $p$ is the mean pressure, $\boldsymbol{f}$ is the vector of mean body forces, $v$ is the kinematic viscosity, $\boldsymbol{S}(\boldsymbol{u})$ is the symmetrical part of the velocity gradient and

$$
\tau=-\overline{\boldsymbol{u}^{\prime} \otimes \boldsymbol{u}^{\prime}}
$$

is the Reynolds stress tensor $\left(\boldsymbol{u}^{\prime}\right.$ is the fluctuating velocity and the overbar denotes the Reynolds average). The boundary conditions for problem (1)-(2) considered are

$$
\begin{aligned}
& \boldsymbol{u}=\overline{\boldsymbol{u}} \quad \text { on } \quad \Gamma_{\mathrm{du}}, \\
& \boldsymbol{n} \cdot \boldsymbol{\sigma}=\overline{\boldsymbol{t}} \quad \text { on } \quad \Gamma_{\mathrm{nu}}, \\
& \boldsymbol{u} \cdot \boldsymbol{n}=\bar{u}_{n}, \quad \boldsymbol{n} \cdot \boldsymbol{\sigma} \cdot \boldsymbol{g}_{1}=\bar{t}_{1}, \quad \boldsymbol{n} \cdot \boldsymbol{\sigma} \cdot \boldsymbol{g}_{2}=\bar{t}_{2} \quad \text { on } \quad \Gamma_{\mathrm{mu}},
\end{aligned}
$$

where $\boldsymbol{\sigma}$ is the Cauchy stress tensor and $\boldsymbol{n}$ is the unit exterior normal to $\partial \Omega$. This boundary $\partial \Omega$ has been considered split into three sets of disjoint components $\Gamma_{\mathrm{du}}, \Gamma_{\mathrm{nu}}$ and $\Gamma_{\mathrm{mu}}$, the latter being the part of the boundary where mixed conditions are prescribed: the normal velocity and the tangent stresses. Vectors $g_{1}$ and $g_{2}$ (for the three-dimensional case) span the space tangent to $\Gamma_{\mathrm{mu}}$. In the classical wall law prescriptions for turbulent flows, the components of the traction vector $\bar{t}_{1}$ and $\vec{t}_{2}$ are expressed in terms of the velocity, thus introducing a non-linearity in the boundary conditions. The simplest of these wall laws is described later on.

The Reynolds stress tensor $\tau$ requires modelling. There are two possibilities that shall be considered in what follows. The first of them (and the simplest) is the Boussinesq assumption and the second is the use of algebraic stress models (ASM).

The Boussinesq assumption consists in taking $\tau$ as
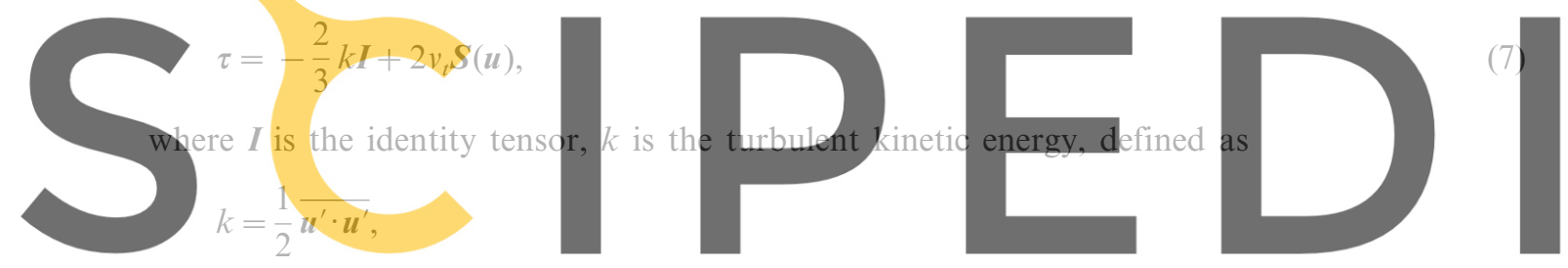

and $v_{t}$ is the so-called turbulent (kinematic) viscosity. Different turbulence models are obtained

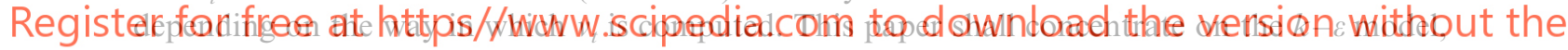

in which

$$
v_{t}=c_{\mu} \frac{k^{2}}{\varepsilon} .
$$

In this equation, $c_{\mu}$ is a constant that is normally taken as $c_{\mu}=0.09$, and $\varepsilon$ is rate of turbulent energy dissipation.

If assumption (7) is used, the Navier-Stokes equations (1) and (2) can be written in terms of a total viscosity $v+v_{t}$. Inserting the expression of $\tau$ in (7) into (1) yields

$$
(\boldsymbol{u} \cdot \nabla) \boldsymbol{u}-\nabla \cdot\left[2\left(v+v_{t}\right) \boldsymbol{S}(\boldsymbol{u})\right]+\nabla p^{*}=\boldsymbol{f},
$$

where the volumetric part of $\tau$ has been included in the pressure term:

$$
p^{*}=p+\frac{2}{3} k
$$

Another possibility is using $p$ instead of $p^{*}$ and modifying the external force $f$ to 


$$
f^{*}=f-\frac{2}{3} \nabla k
$$

The authors have used this last possibility in the numerical examples since using $p^{*}$ it is not possible to prescribe boundary conditions involving the physical pressure $p$, such as in the case in which the traction is prescribed. In what follows, the superscript $*$ in $\boldsymbol{f}$ shall be omitted and it should be considered that when the $k-\varepsilon$ model is used, the viscosity must be replaced by $v+v_{t}$.

\subsection{Equations of the $k-\varepsilon$ model}

The equations for $k$ and $\varepsilon$ have also to be modelled, which means that several assumptions have to be made in order to close the problem, since these equations involve moments of $\boldsymbol{u}^{\prime}$ of order higher than two. In the $k-\varepsilon$ model, the differential equations for the turbulent kinetic energy $k$ and the rate of turbulent energy dissipation $\varepsilon$ to be solved are $[2,3]$

$$
\begin{aligned}
& (\boldsymbol{u} \cdot \nabla) \varepsilon-\nabla \cdot\left(\frac{v_{t}}{\sigma_{\varepsilon}} \nabla \varepsilon\right)-\frac{\varepsilon}{k}\left(C_{1} P_{k}-C_{2} \varepsilon\right)=0, \\
& (\boldsymbol{u} \cdot \nabla) k-\nabla \cdot\left(\frac{v_{t}}{\sigma_{k}} \nabla k\right)-P_{k}+\varepsilon=0, \\
& P_{k}=2 v_{t} S(\boldsymbol{u}): S(\boldsymbol{u}),
\end{aligned}
$$

where

is the so-called production term and $\sigma_{k}=1.0, \sigma_{\varepsilon}=1.3, C_{1}=1.44$ and $C_{2}=1.92$ are experimental constants. In Equation (11), the colon stands for the double contraction of second-order tensors. In Equations (9) and for $k$ and $\varepsilon$ has been neglected. (e), and $v_{t} / \sigma_{k}$ by $v+v_{t} / \sigma_{k}$ in (10) Proper boundary conditions have brief comment on them will f prescribed (Dirichlet-type of boundary condition). The

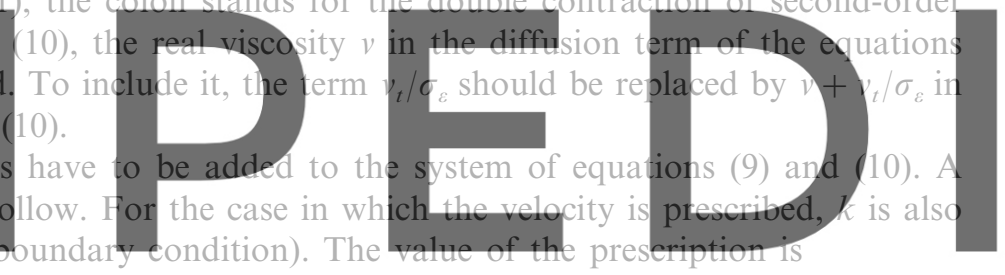

Register for free at https//www.scipedia.com to download the version without the where $c_{\mathrm{bc}}$ is a constant and $u$ is the Euclidean norm of the velocity. The constant $c_{\mathrm{bc}}$ is taken as approximately between 0.003 and 0.01 . When $k$ is prescribed, so is $\varepsilon$. The prescription for it is

$$
\varepsilon=c_{\mu} \frac{k^{3 / 2}}{L}
$$

where $L$ is again a constant, the physical meaning of which is the characteristic length of the model. On the boundary it must be given, but in the interior of the computational domain, the characteristic length (or mixing length) for the $k-\varepsilon$ model is

$$
L=c_{\mu} \frac{k^{3 / 2}}{\varepsilon},
$$

so that $v_{t}=\sqrt{k} L$.

Equations (12) and (13) give the boundary conditions on the part of the boundary where $\boldsymbol{u}$ is prescribed, i.e. on $\Gamma_{\mathrm{du}}$. On $\Gamma_{\mathrm{nu}}$, the boundary conditions taken are 


$$
\frac{\partial k}{\partial n}=0, \quad \frac{\partial \varepsilon}{\partial n}=0
$$

where $\partial / \partial n$ is the normal derivative on the boundary of $\Omega$. The same boundary condition is prescribed on $\Gamma_{\mathrm{mu}}$ except when a wall law is prescribed there. In this case, the traction on the boundary is given by

$$
\boldsymbol{t}=-\rho \frac{U_{*}^{2}}{|\boldsymbol{u}|} \boldsymbol{u},
$$

where $\rho$ is the fluid density and $U_{*}$ is the solution of the non-linear equation

$$
\frac{|\boldsymbol{u}|}{U_{*}}=\frac{1}{\kappa} \log \left(\frac{U_{*} \Delta}{v}\right)+C,
$$

with $\kappa=0.41$ (von Kármán constant), $C=5.5$, and where $\Delta$ is the distance from the wall at which the velocity is evaluated.

When a wall law is prescribed for the velocity (i.e. for the Navier-Stokes equations), $k$ and $\varepsilon$ are prescribed to

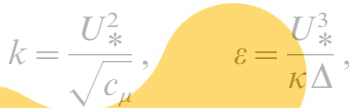

and therefore $\Gamma_{\mathrm{mu}}$ is a part of the boundary where Dirichlet boundary conditions are also prescribed for $k$ and $\varepsilon$.

This completes the definition of the $k-\varepsilon$ turbulence model. Equations (1) and (2) and (9) and (10) have to be solved and Equation (7) (with $v_{t}$ given by (8)) is used to compute the Reynolds

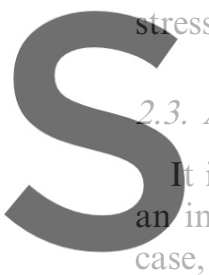

An algebraic stress model
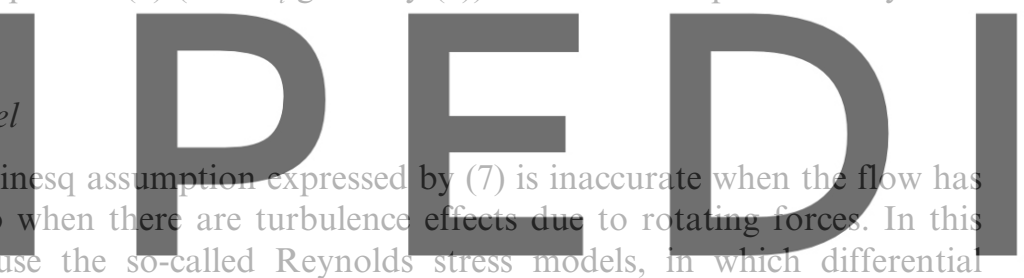

equations are proposed to model the components of the Reynolds stress tensor [14]. These

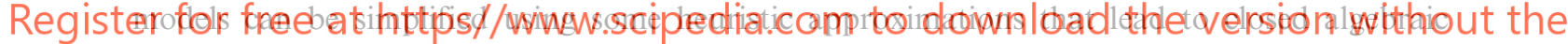

expressions for the components of $\tau[3,14]$. This is the basis of the ASMs, in which the tensor

$\tau$ is expressed in terms of $k$ and $\varepsilon$, so that the solution of (9) and (10) is again needed.

The ASM that shall be considered in one of the numerical examples in Section 6 is the regularized version of the models presented in [13]. This model in particular is designed in order to take into account the effects of Coriolis and centrifugal forces, i.e. the equations of motion can be formulated in a reference system rotating with an angular velocity vector $\omega$. In this case, the Coriolis force $2 \omega \times \boldsymbol{u}$ and the centrifugal force $\omega \times(\omega \times \boldsymbol{r})$ ( $\boldsymbol{r}$ being the vector of position of the particles) have to be added to the left-hand-side of (1).

In contrast to other ASM, in this case the Reynolds stress tensor is given explicitly, without the need of solving an implicit non-linear equation for its components (e.g. the ASM presented in $[3,12,14])$. This model is given by

$$
\frac{\tau_{i j}}{\rho}=\frac{6\left(1+\eta^{2}\right) \alpha_{1} k}{3+\eta^{2}+6 \zeta^{2} \eta^{2}+6 \zeta^{2}}\left[S_{i j}^{*}+\left(S_{i k}^{*} W_{k j}^{*}+S_{j k}^{*} W_{k i}^{*}\right)-2\left(S_{i k}^{*} S_{k j}^{*}-\frac{1}{3} S_{k l}^{*} S_{k l}^{*} \delta_{i j}\right)\right],
$$

where $\alpha_{1}=\left(C_{2}-\frac{4}{3}\right) /\left(C_{3}-2\right)$ and 


$$
\begin{aligned}
& S_{i j}^{*}=\frac{1}{2} g \tau\left(2-C_{3}\right) S_{i j}, \\
& W_{i j}^{*}=\frac{1}{2} g \tau\left(2-C_{4}\right)\left[W_{i j}+\left(\frac{C_{4}-4}{C_{4}-2}\right) e_{m j i} \omega_{m}\right], \\
& \eta=\left(S_{i j}^{*} S_{i j}^{*}\right), \quad \zeta=\left(W_{i j}^{*} W_{i j}^{*}\right),
\end{aligned}
$$

and

$$
g=\left(\frac{1}{2} C_{1}+\frac{P_{k}}{\varepsilon}-1\right)^{-1}, \quad \tau=\frac{k}{\varepsilon} .
$$

In these equations, $P_{k}$ is the production term defined in (11), $\omega_{m}$ is the $m$ th component of the speed of rotation vector, $e_{m j i}$ are the components of the permutation tensor and $W_{i j}$ has been used for the components of the skew-symmetric part of the velocity gradient.

The physical constants appearing in this turbulence model are given by (see [13])

$$
C_{1}=6.80, \quad C_{2}=0.36, \quad C_{3}=1.25, \quad C_{4}=0.40 \text {. }
$$

Although Equation (19) describes a particular case of an ASM, the iterative strategy presented in the following section provides a general framework for the implementation of other ASMs. The expression for the Reynolds stress $\tau$ will be assumed to be given, and the iterative technique will be described in terms of it.

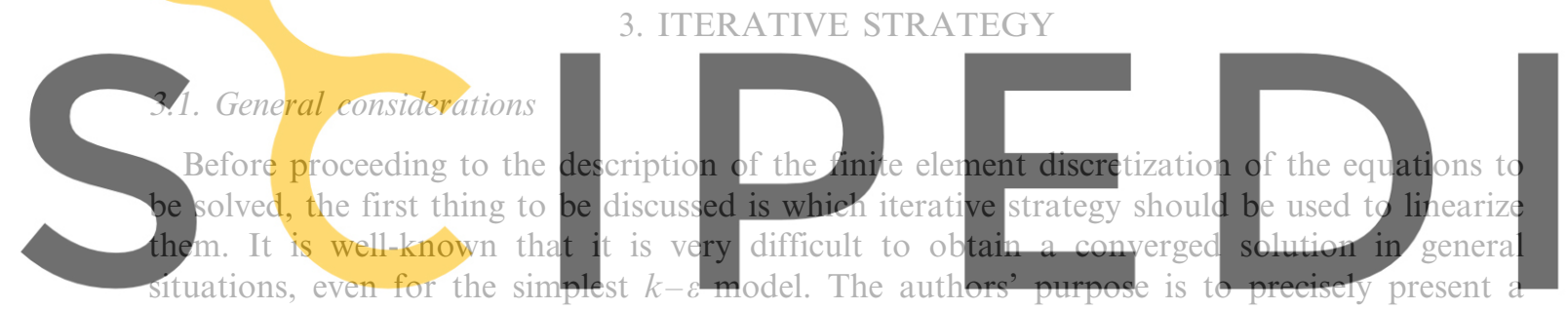

technique that they have found very robust in many numerical examples.

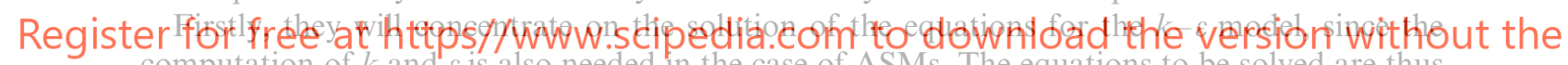
computation of $k$ and $\varepsilon$ is also needed in the case of ASMs. The equations to be solved are thus

(1) and (2) and (9) and (10), together with the boundary conditions described in the previous section.

First of all, Equations (9) and (10) must be linearized. They form a coupled system of equations that is in turn coupled with the Navier-Stokes equations for the mean velocity $\boldsymbol{u}$. The strategy followed is to solve for $\boldsymbol{u}$ and then to solve for $k$ and $\varepsilon$ until convergence, repeating the process if needed.

The scheme is as follows:

1. Solve the Navier-Stokes equations.

2. Solve for $k$ and $\varepsilon$

2.1. Update production

2.2. Solve for $k$ (until convergence)

2.3. Solve for $\varepsilon$ (until convergence)

2.4. Check convergence in terms of $L$. If not, go to 2.2 .

3. Check convergence for $\boldsymbol{u}$. If not, go to 1 . 
Concerning the linearization of the Navier-Stokes equations (1) and (2), taken into account is the possibility of using either a fixed point (or Picard-type) linearization as well as a Newton-Raphson one. The latter is faster, but the former is slightly cheaper and, what is more important, more robust. Also, there is the possibility of using an underrelaxation parameter to improve the speed of convergence.

The details of this iterative scheme are given in the following subsections.

\subsection{Linearization of the equations for $k$ and $\varepsilon$}

First consider a convection-diffusion-production equation of the form

$$
(\boldsymbol{u} \cdot \nabla) \phi-\nabla \cdot(\kappa \nabla \phi)+\alpha \phi=f,
$$

where $\kappa$ and $\alpha$ are constants and $f$ is a known source term. It is known that this equation is 'well-behaved' when $\kappa>0$ and $\alpha>0$ (or both are negative). In this case, the bilinear form associated to the problem (see below) is coercive (in the simple case $\phi=0$ on $\partial \Omega$ ) and the finite element approximation to the problem is in principle possible for any value of $\kappa$ and $\varepsilon$. However, if any of these coefficients are negative this property can be lost depending on their relative magnitude. This is the main idea for the following linearization of the equations for $k$ and $\varepsilon$.

Suppose, given velocity field $\boldsymbol{u}$, and you have to compute $k$ and $\varepsilon$ with this velocity.

The first point of the iterative scheme is that the equations for $k$ and $\varepsilon$ are coupled iteratively. Thus, the non-linear equation is solved for $k$ assuming that $\varepsilon$ is known and then the computed value of $k$ is used to solve the non-linear equation for $\varepsilon$. Since only updated values are used, this technique can be viewed as a block Gauss-Seidel iterative coupling [15].
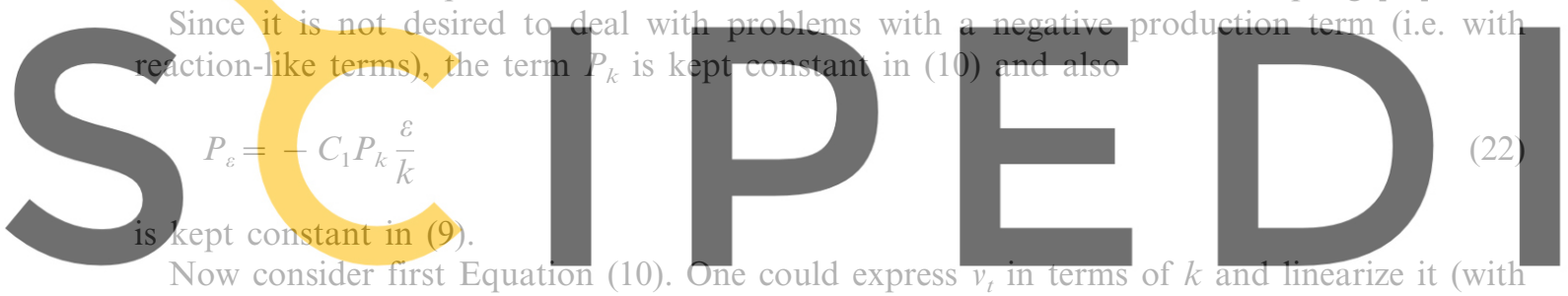

$\varepsilon$ given). However, $v_{t}$ is kept constant while iterating for $k$ to avoid the possibility of having a

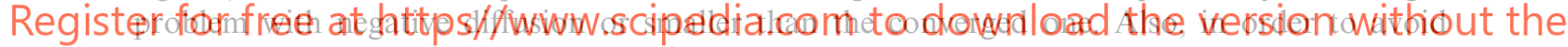

keeping $\varepsilon$ constant, it is written as $c_{\mu} k^{2} / v_{t}$ and linearized using the Newton-Raphson method.

Therefore, the innermost iterative loop to be performed is:

Given $v_{t}$ and $P_{k}$, solve until convergence:

$$
(\boldsymbol{u} \cdot \nabla) k_{i}-\nabla \cdot\left(\frac{v_{t}}{\sigma_{k}} \nabla k_{i}\right)-P_{k}+\frac{c_{\mu}}{v_{t}}\left(2 k_{i-1} k_{i}-k_{i-1}^{2}\right)=0,
$$

where the subscript stands for the iteration counter.

Similarly, $v_{t}$ is also kept constant for (9), so that the linearized version of this equation is:

Given $k, v_{t}$ and $P_{\varepsilon}$, solve until convergence:

$$
(\boldsymbol{u} \cdot \nabla) \varepsilon_{i}-\nabla \cdot\left(\frac{v_{t}}{\sigma_{\varepsilon}} \nabla \varepsilon_{i}\right)-P_{\varepsilon}+\frac{C_{2}}{k}\left(2 \varepsilon_{i-1} \varepsilon_{i}-\varepsilon_{i-1}^{2}\right)=0 .
$$

It is observed that Equations (23) and (24) have the same structure. They are of the general form of (21) with both $\kappa$ and $\alpha$ positive, if it is assumed that each iterate of $k$ and $\varepsilon$ is positive (as they should). 
In the $k-\omega$ model, the two variables that are used to compute the turbulent kinematic viscosity are $k$ and the dissipation per unit of kinetic energy, $\omega$. Different forms of the equation for this last variable can be found in the literature, as discussed in [16]. The authors have implemented the equation proposed in [17] and described also in the previous reference, which reads

$$
(\boldsymbol{u} \cdot \nabla) \omega-\nabla \cdot\left(\frac{v_{t}}{\sigma_{\omega}} \nabla \omega\right)-\frac{\omega}{k}\left(C_{\omega 1} P_{k}-C_{\omega 2} k \omega\right)=0,
$$

where $\sigma_{\omega}=2.0, C_{\omega 1}=5 / 9$ and $C_{\omega 2}=3 / 40$ are empirical constants of the model. Once $k$ and $\omega$ are computed, $\varepsilon$ can be found from the equation

$$
\varepsilon=c_{\mu} \omega k,
$$

and then one can proceed as in the $k-\varepsilon$ model.

Referring to the $k-k \tau$ model proposed in [18] (and also described in [16]), the two variables used are $k$ and an integral time scale $\tau$, although the equations of the model are formulated in terms of $k$ and $k \tau$. The equation for this last variable is

$$
(\boldsymbol{u} \cdot \nabla)(k \tau)-\nabla \cdot\left(\frac{v_{t}}{\sigma_{\tau}} \nabla(k \tau)\right)-\frac{(k \tau)}{k} C_{\tau 1} P_{k}+C_{\tau 2} k=0,
$$

where now $\sigma_{\tau}=10.8, C_{\tau 1}=0.173$ and $C_{\tau 2}=0.225$ are the empirical constants of the model. From $k$ and $\tau$, one can compute $\varepsilon$ from the equation
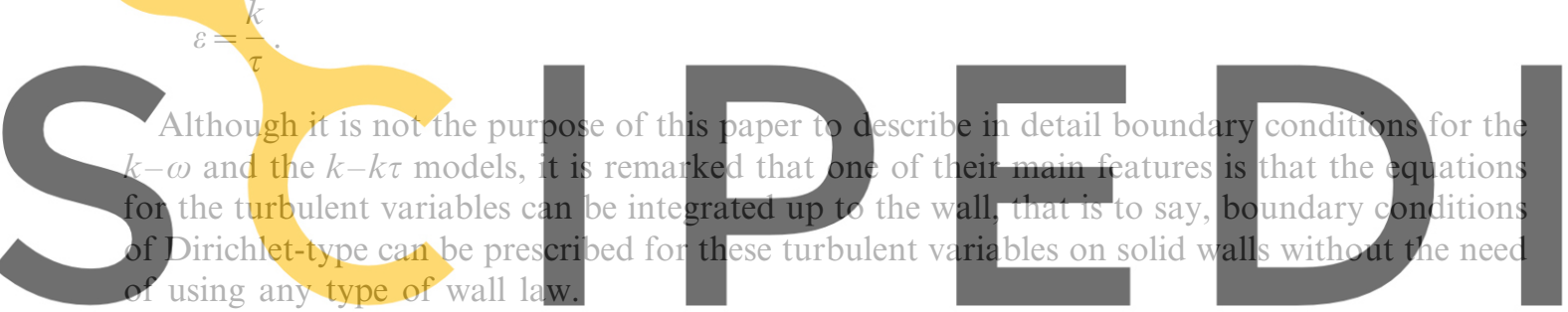

From the point of view of the iterative scheme to be used to solve either (27) or (28), it is

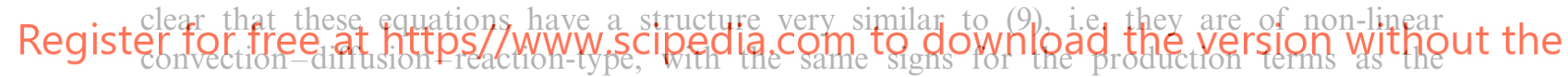

equation for $\varepsilon$. Therefore, exactly the same strategy is used as described above, namely, the scheme presented in Section 3.3 replacing \& either by $\omega$ or by $k \tau$.

In the following section, the authors shall present the finite element approximation of the linearized equations for $k$ and $\varepsilon$. Since the linearized equations for $\omega$ and $\tau$ are very similar to the equation for $\varepsilon$, exactly the same procedures as used for this can be applied for them.

\section{FINITE ELEMENT APPROXIMATION}

\subsection{Model problem and basic formulation}

Consider first the finite element approximation of the equations for $k$ and $\varepsilon$. Once they have been linearized, both can be considered as convection-diffusion-production equations, i.e. they are of the form indicated in (21). Now, the basic finite element formulation that is used to approximate these type of equations numerically is described. 
tion and to reaction, is presented. For consistency, the new dissipation added must be proportional to the element residual and, for accuracy, it must vanish quickly in regions where the solution is smooth. The expression of the numerical dissipation presented in [5] is based on a simple study of the discrete maximum principle.

The idea is to introduce a non-linear numerical scheme in which the diffusion added is given by

$$
\kappa_{\mathrm{dc}}=\frac{1}{2} \xi_{\mathrm{c}} h \frac{\left|\mathscr{R}\left(\phi_{h}\right)\right|}{\left|\nabla \phi_{h}\right|} \text { for each element, }
$$

where the parameter $\xi_{c}$ is computed as

$$
\xi_{\mathrm{c}}=\max \left\{0, C_{\mathrm{dc}}-\frac{2 \kappa}{\left|\boldsymbol{u}^{*}\right| h}\right\}
$$

with

$$
\boldsymbol{u}^{*}:=\frac{1}{\left|\nabla \phi_{h}\right|^{2}}\left(\boldsymbol{u} \cdot \nabla \phi_{h}+\sigma \phi_{h}-f\right) \nabla \phi_{h},
$$

and $C_{\mathrm{dc}}$ is a constant. From numerical experiments, it has been found that an effective choice is to take $C_{\mathrm{dc}}=0.7$ for linear elements and $C_{\mathrm{dc}}=0.35$ for quadratics.

The problem now is to decide in which directions is it necessary to introduce the diffusion $\kappa_{\mathrm{dc}}$. It can be readily seen that the numerical diffusion inherent to the SUPG formulation acting only along the streamlines is given by

$$
\kappa_{\mathrm{SUPG}}=\frac{1}{2} \xi h|\boldsymbol{u}|
$$

which may very well be greater than $\kappa_{\mathrm{dc}}$. Therefore, the numerical dissipation that has to be introduced along the streamlines is only

$$
\kappa_{\mathrm{sl}}=\max \left\{0, \kappa_{\mathrm{dc}}-\kappa_{\mathrm{SUPG}}\right\} .
$$

After this heuristic reasoning, we are led to the introduction of a non-linear anisotropic diffusion to the basic numerical formulation given by (31). The final numerical method will consist of the addition of the term

$$
\sum_{e=1}^{n_{\mathrm{el}}} \int_{\Omega^{e}}\left[\kappa_{\mathrm{dc}} \nabla \psi_{h} \cdot \nabla \phi_{h}+\left(\kappa_{\mathrm{sl}}-\kappa_{\mathrm{dc}}\right) \nabla \psi_{h} \cdot\left(\frac{1}{|\boldsymbol{u}|^{2}} \boldsymbol{u} \otimes \boldsymbol{u}\right) \cdot \nabla \phi_{h}\right] \mathrm{d} \Omega
$$

to the left-hand-side of (31).

Using different ideas, similar methods have been proposed before [21-23]. The main difference between this approach and previous methods is the consideration of the diffusion associated to the SUPG method, which leads to the second term in (38).

It is shown in [4,5] for the diffusion-convection equation that the method proposed here has two important advantages with respect to the introduction of an isotropic non-linear diffusion. First, it is much less diffusive and, what is more important, it has much better convergence properties. All these aspects are treated in more detail in the above mentioned references.

Although this paper has been restricted to the case of linear elements, similar conclusions are valid for quadratic ones. 


\subsection{Comments on the approximation of the Navier-Stokes equations}

The finite element approximation of the incompressible Navier-Stokes equations (1) and (2) is standard and therefore it is only presented briefly.

For the finite element interpolation of the velocity and the pressure, mixed interpolations satisfying the so-called Babuska-Brezzi (BB) stability condition [24] have been used. In particular, for the examples presented later on two types of interpolation have been used: the $Q_{1} / P_{0}$ and the $Q_{2} / P_{1}$ elements. The former consists of multilinear continuous velocities and piecewise constant discontinuous pressures. This element does not satisfy strictly the BB condition but is known to yield good results for most of the cases. The $Q_{2} / P_{1}$ element is constructed using multiquadratic continuous velocities and piecewise linear discontionuous pressures.

When the pressure interpolation used is discontinuous (as in the cases described above) it is possible to eliminate the pressure degrees of freedom at the element level using a penalty strategy. This is done using the iterative penalty method presented in [25].

The discretization of the incompressibility constraint leads to an equation of the form

$$
\mathrm{DU}=\mathbf{0},
$$

where $\boldsymbol{D}$ is the discrete divergence matrix and $\boldsymbol{U}$ is the vector of nodal velocities. Instead of using the standard penalty method, the following modification of the previous equation was used:

$$
\varepsilon \boldsymbol{M}_{\mathrm{p}} \boldsymbol{P}_{i}+\boldsymbol{D} \boldsymbol{U}_{i}=\varepsilon \boldsymbol{M}_{\mathrm{p}} \boldsymbol{P}_{i-1},
$$

where $\varepsilon$ is a small number (penalty parameter), $\boldsymbol{M}_{\mathrm{p}}$ is the pressure mass matrix, $\boldsymbol{P}$ is the vector of nodal pressures and the subscript is the iteration counter. This method is described and fully analyzed in [25]. The incompressibility restriction is iteratively approximated as the iterative procedure goes on, thus allowing the use of penalty parameters larger than using the classical penalty method.

Finally, in order to be able to deal with flows with a high element Reynolds number, the SUPG method is also used for the Navier-Stokes equations. The formulation is similar to that described in the previous section for (21). Once this is done, the final discrete algebraic version of the momentum equation at each iteration will be of the form

$$
\boldsymbol{K}_{i-1} \boldsymbol{U}_{i}+\boldsymbol{G P}_{i}=\boldsymbol{u} \boldsymbol{F}_{i-1} .
$$

Here, $\boldsymbol{G}$ is the discrete gradient matrix and matrix $\boldsymbol{K}_{i-1}$, accounting for both viscous and convective terms, depends on the velocity unknowns $\boldsymbol{U}_{i-1}$ through the linealization of the convective terms and the Reynolds stress. Vector $\boldsymbol{F}_{i-1}$ in (41) depends also on $\boldsymbol{U}_{i-1}$ through these linearizations and also through the law of the wall given by (16), with the normal component of the velocity prescribed to zero. To do this, it is necessary to construct a basis with one vector normal to the boundary and the others tangent to it, refer the velocity to this basis and impose the prescription in it.

\section{NUMERICAL EXAMPLES}

\subsection{Flow over a backward-facing step}

This is one of the standard benchmark problems for turbulent flows. The computational domain is the rectangle $[0,22] \times[0,1.5]$, with a step of length 3 and height 0.5 at the bottom 
left corner. This domain has been discretized using a rather coarse finite element mesh of 1632 $Q_{1} / P_{0}$ elements (bilinear velocities, piecewise constant pressures) and 1721 nodal points, as well as a mesh of $Q_{2} / P_{1}$ elements (biquadratic velocities, piecewise linear pressures) with the same nodes as the former. In both cases, the SUPG method and the discontinuity capturing technique for the spatial discretization has been employed.

As boundary conditions, a constant velocity $(1,0)$ has been prescribed at the inlet $(x=0)$, whereas at the outlet $(x=22)$, the horizontal velocity has been left free (zero normal traction) and the vertical one has been prescribed to zero. On the rest of the boundary, the normal velocity has been prescribed to zero and a wall law has been used to compute the tangent stress, with $\Delta=0.05$ (see (17)). As for the boundary conditions for the turbulent parameters, Equations (12) and (13) have been used with $c_{\mathrm{bc}}=0.003$ and $L=0.03$ for the inlet, (15) for the outlet and (18) for the rest of the boundary. The Reynolds number based on the inlet velocity and the height of the step, $H$, is 70000 .

For this example, there are many numerical and experimental results (see e.g. [26]). The experimental reattachment length is found to be $(7.0 \pm 1.0) H$, whereas numerical results give values around $6 \mathrm{H}$. The authors have obtained a reattachment length of $6.57 \mathrm{H}$, both using the $Q_{1} / P_{0}$ and the $Q_{2} / P_{1}$ elements (see Figure 1).

The authors interest in this study is to have an indication of the convergence behaviour of the algorithm. For that, they have plotted in Figure 2 the convergence of the velocity, i.e. the outermost loop of the iterative scheme described in Section 3. Figures 3 and 4 plot the convergence of $L$ for the first iteration for $\boldsymbol{u}$ (intermediate loop) and the convergence of $k$ and $\varepsilon$ for the first iteration of $L$ (innermost loop). Both cases are the worst of the whole process, since less iterations of $L$ and of $k$ and $\varepsilon$ are needed as the velocity converges. It is seen from the previous figures that the behaviour of the scheme is similar for both the $Q_{1} / P_{0}$ and the $Q_{2} / P_{1}$ elements. In all the cases, the residual is measured using the Euclidean norm of the difference of the unknown being considered between the current and the previous iterate, and this is divided by the Euclidean norm of the previous iterate.

\subsection{Flow in a rotating polar cavity}

Now, the numerical results obtained for the flow in a rotating polar cavity are presented. The domain is a sector of $45^{\circ}$ delimited by two circumferences of radius 1 and 2 rotating with an angular velocity $\omega$ normal to the plane where the domain is and with norm 1 .

On the outer circumference a tangent velocity of modulus 2 has been prescribed. There, $k$ and $\varepsilon$ have been fixed to $k=0.003$ and $\varepsilon=0.0004295$, which corresponds to $L=0.03$. On the

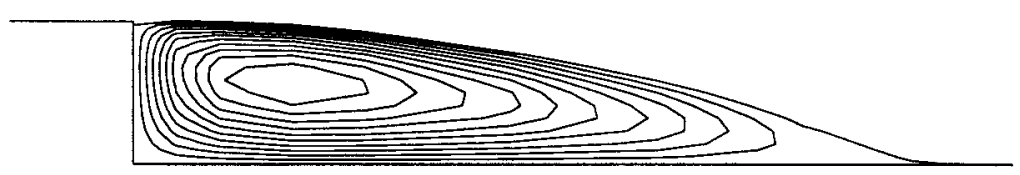

Figure 1. Detail of the streamlines obtained using the $Q_{1} / P_{0}$ element. 


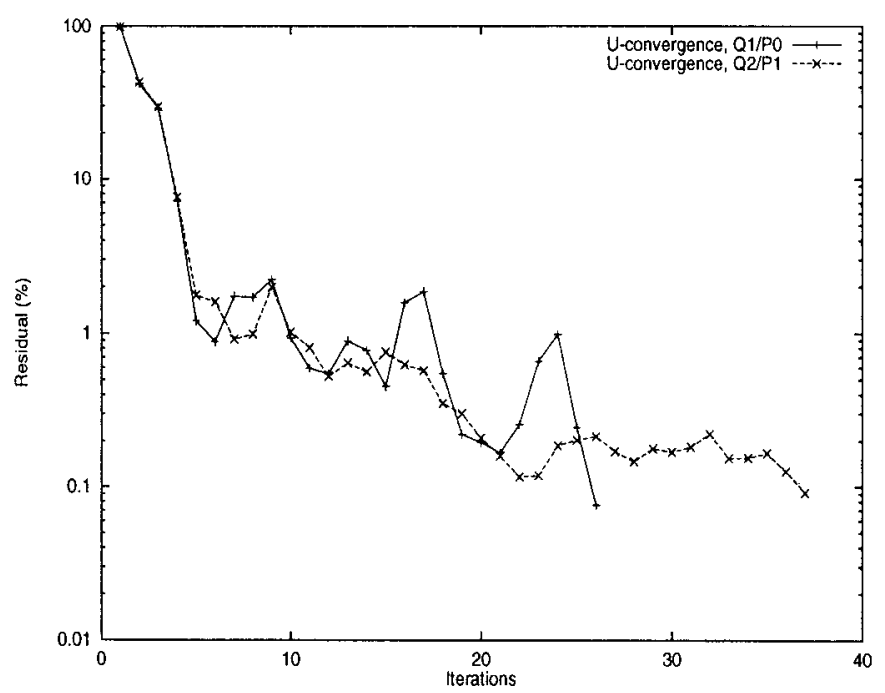

Figure 2. Velocity convergence for the flow over a backward-facing step.

rest of the boundary, the prescriptions are zero normal velocities and tangent stress given by the wall law with $\Delta=0.05$. The kinematic viscosity of the fluid has been taken as $v=5 \times 10^{-8}$, which gives a Reynolds number of $4 \times 10^{8}$.

Figure 5 plots the streamlines and the pressure contours obtained using the standard $k-\varepsilon$ model and the ASM described in Section 2.3. The latter captures two (weak) vortices at the top and bottom left corners of the cavity that are not obtained using the $k-\varepsilon$ model. A detail of what happens in the bottom left corner is plotted in Figure 6.

The values of $k$ and $\varepsilon$ introduced by the $k-\varepsilon$ model are higher than those predicted by the ASM, as can be seen from Figure 7. This yields a flow in which the Reynolds stresses dominate and the effects of rotation are hidden. In this case, the numerical solution is independent of the

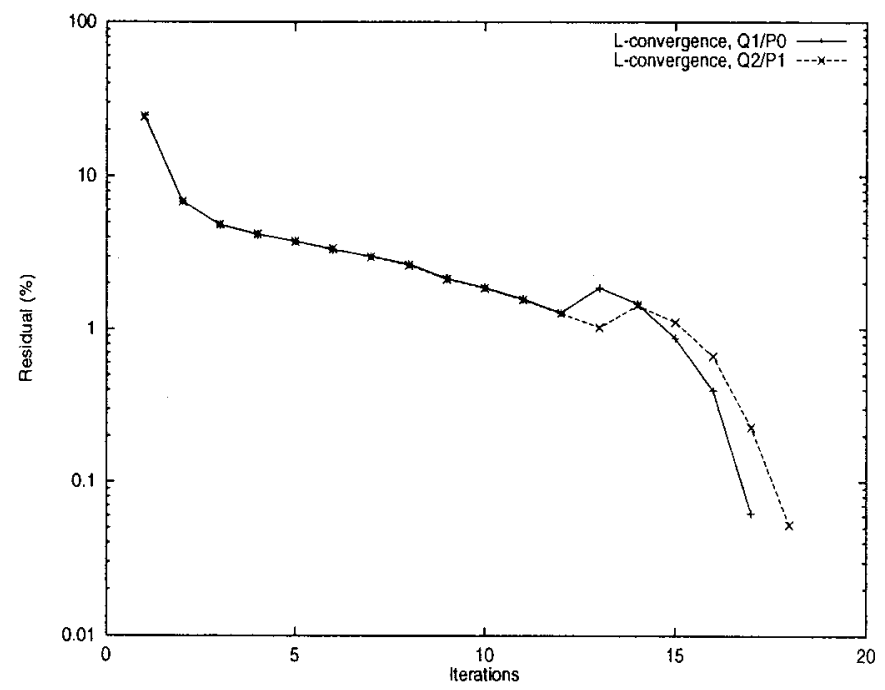

Figure 3. Convergence of $L$ for the flow over a backward facing step. First iteration of $\boldsymbol{u}$. 


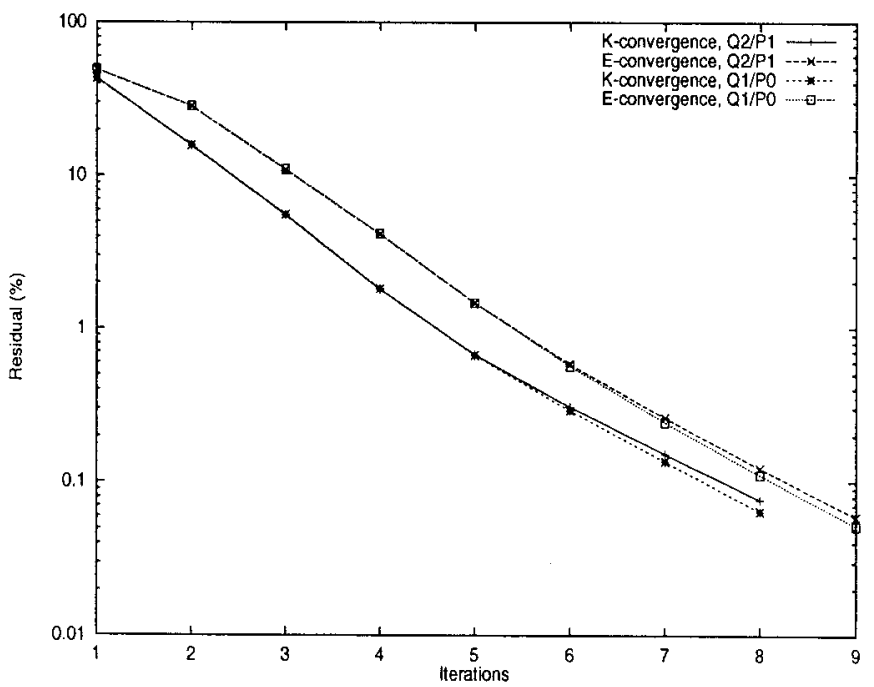

Figure 4. Convergence of $k$ and $\varepsilon$ for the flow over a backward-facing step. First iteration of $\boldsymbol{u}$ and first iteration of $L$.

speed of rotation $\omega$, which does not happen using the ASM. From the pressure contours in Figure 5 it is observed that the pressure is basically determined by the centrifugal force in this case.
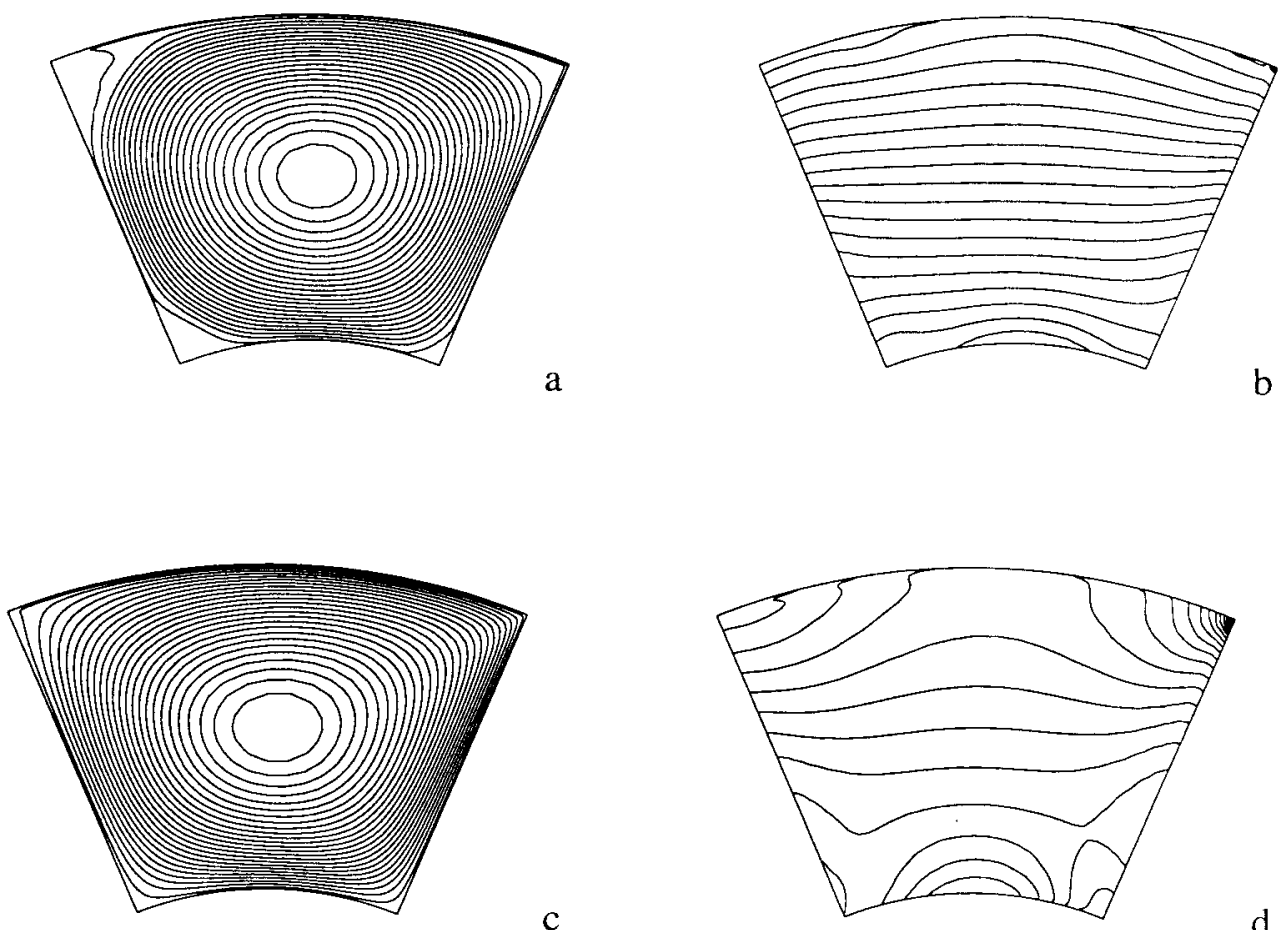

Figure 5. (a) Streamlines using the ASM. (b) Pressure contours using the ASM. (c) Streamlines using the $k-\varepsilon$ model. (d) Pressure contours using the $k-\varepsilon$ model. 

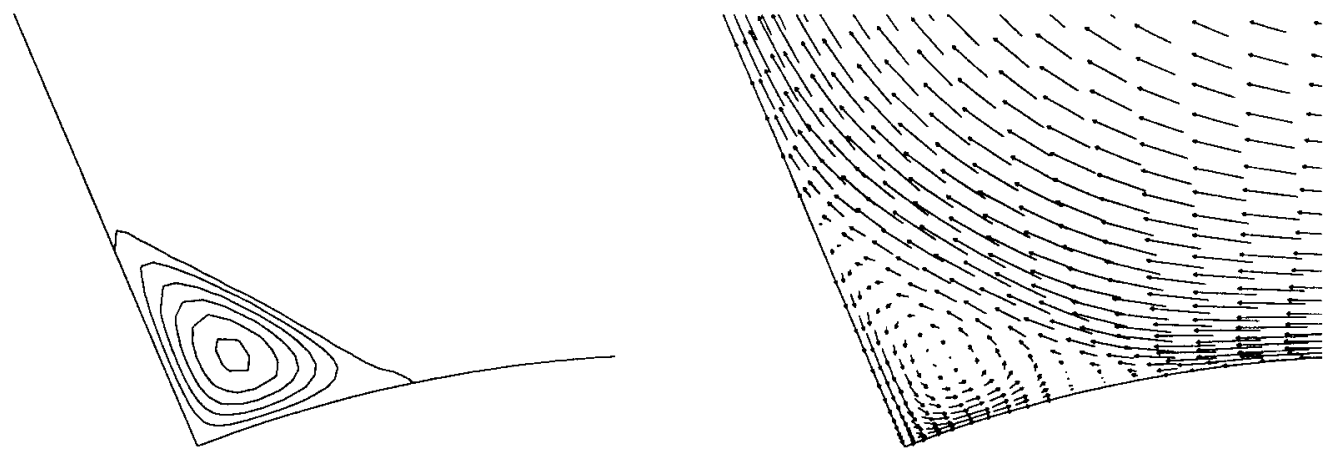

a
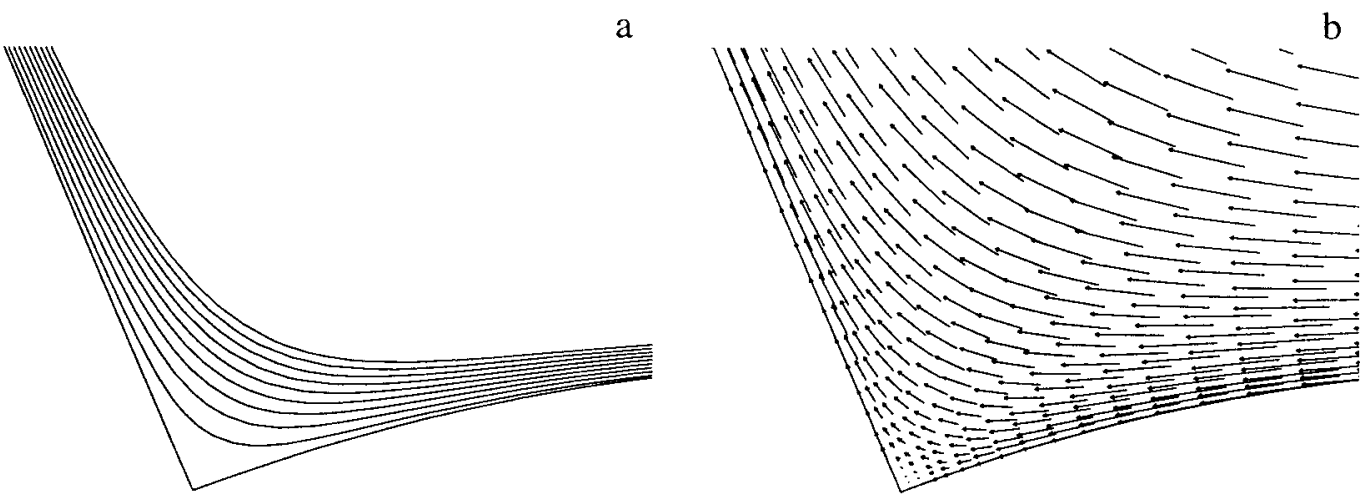

$\mathrm{c}$

Figure 6. (a) Detail of the streamlines using the ASM. (b) Detail of the velocity vectors using the ASM. (c) Detail of the streamlines using the $k-\varepsilon$ model. (d) Detail of the velocity vectors using the $k-\varepsilon$ model.

Referring to the convergence behaviour of the algorithm, Figure 8 plots the velocity convergence using the $k-\varepsilon$ and the ASM. It is observed that the latter is clearly worse. The final residual oscillates around the 1\%. If the preconditioning described in Section 3.4 is not used, the iterative scheme diverges. Nevertheless, the solution obtained using the ASM is smooth, as it can be seen from the previous pictures as well as from Figure 9, where the profiles of $k$ and $\varepsilon$ using the $k-\varepsilon$ and the ASM are shown. Although the numerical strategy and the physical properties for the evaluation of $k$ and $\varepsilon$ are exactly the same for both models, it is remarkable that the ASM yields values of these turbulent variables much smaller that the $k-\varepsilon$ model.

Finally, Figures 10 and 11 plot the convergence of $L$ for the first iteration of $\boldsymbol{u}$ and the convergence of $k$ and $\varepsilon$ for the first iteration of $L$. In this and in other iterations, it turns out that $k$ and $\varepsilon$ converge better using the ASM.

\subsection{Flow over a wing}

It is known that the flow around a body placed on a wall develops a horseshoe upstream vortex at the intersection between the body and the wall. In this example, one of these situations is considered, namely the turbulent flow at the junction of a flat surface and a normally mounted cylindrical wing. The geometry of the computational domain is shown in 

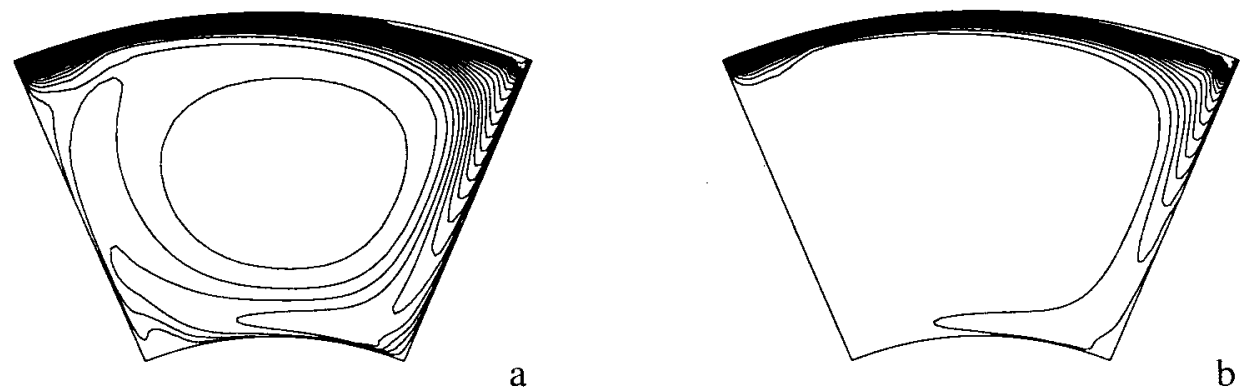

a

b
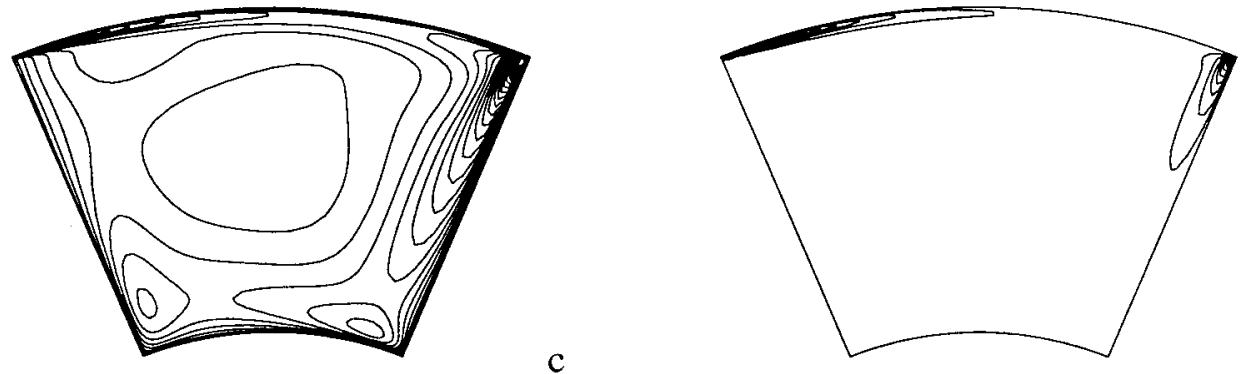

d

Figure 7. (a) Contours of $k$ using the ASM; $\max =0.03$. (b) Contours of $\varepsilon$ using the ASM; $\max =0.05$. (c) Contours of $k$ using the $k-\varepsilon$ model; $\max =0.38$. (d) Contours of $\varepsilon$ using the $k-\varepsilon$ model; $\max =9.25$.

Figure 12 and the experimental velocity pattern in Figure 13. These pictures, as well as the set-up of the whole problem, have been obtained from [27]. As indicated in the information supplied there, the wing has a maximum thickness of $T=71.7 \mathrm{~mm}$ and a chord of $305 \mathrm{~mm}$. It

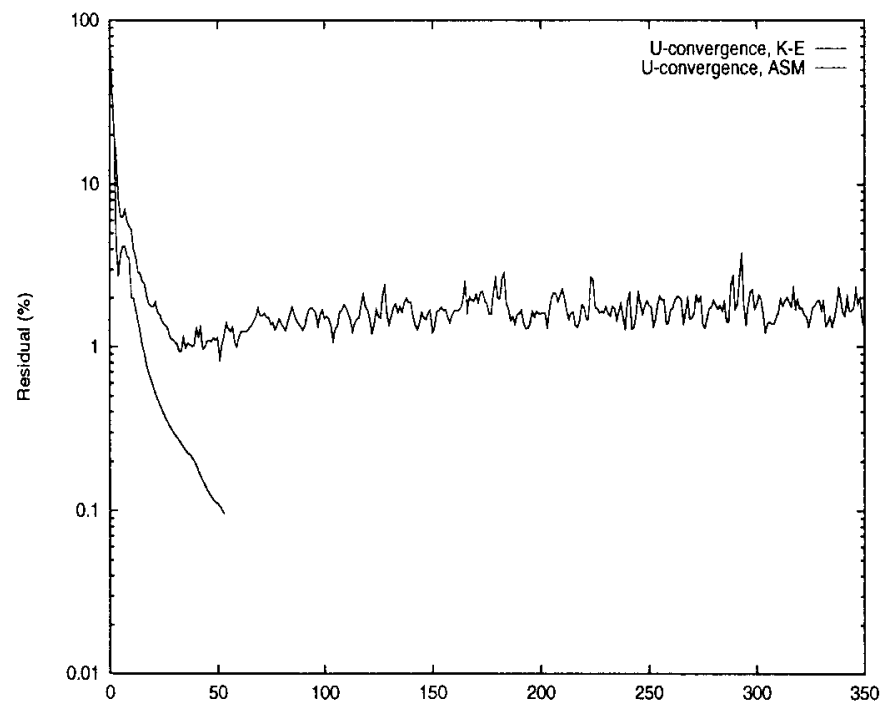

Figure 8 . Velocity convergence for the flow in a polar cavity. 

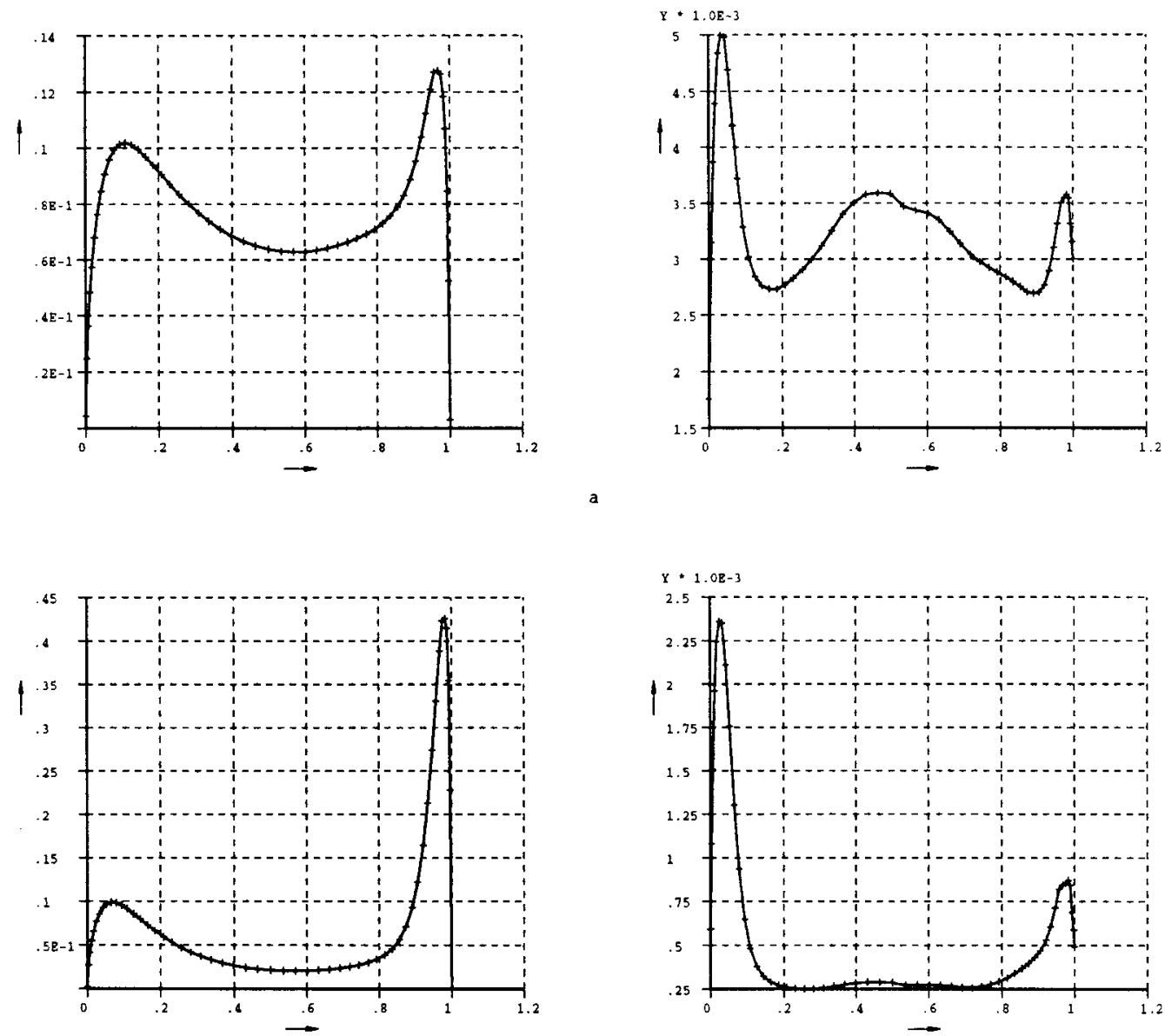

Figure 9. Profiles along the mid-section of the polar cavity. (a) $k$ using the $k-\varepsilon$ model. (b) $k$ using the ASM. (c) $\varepsilon$ using the $k-\varepsilon$ model. (d) $\varepsilon$ using the ASM.

is situated 18.24T downstream from the inlet. Its cross-section consists of a 3:2 elliptical nose and a NACA 0020 tail joined at the maximum thickness. Experimental results for this problem are reported in [28], which were used for validation of the numerical results presented in [29].

Owing to the symmetries of the problem, only the fourth of the domain needs to be modelled. The finite element mesh employed in these calculations is shown in Figure 14. It consists of $37296 Q_{1} / P_{0}$ elements and 41250 nodal points. In order to be able to approximate properly the law of the wall, there is also a very thin layer of elements next to the flat surface. The width of this layer is 1000 times smaller than that of the next layer.

As boundary conditions, the $x$-velocity component has been prescribed at the inlet to 26990 $\mathrm{mm} \mathrm{s}^{-1}$, whereas the $y$ - and $z$-components have been fixed to zero. The kinematic viscosity of the fluid (air) is $16.60802 \mathrm{~mm}^{2} \mathrm{~s}^{-1}$, which gives a Reynolds number based on the thickness $T$ of 116521 . The wall of the law has been used on the boundaries, except for the symmetry planes (where $\partial k / \partial n=0, \partial \varepsilon / \partial n=0$ ) and at the inlet and the outlet. Some numerical results obtained for this problem using $\Delta=2 \mathrm{~mm}$ are plotted in Figure 15 (contours of pressure, $k$ and $\varepsilon)$. 


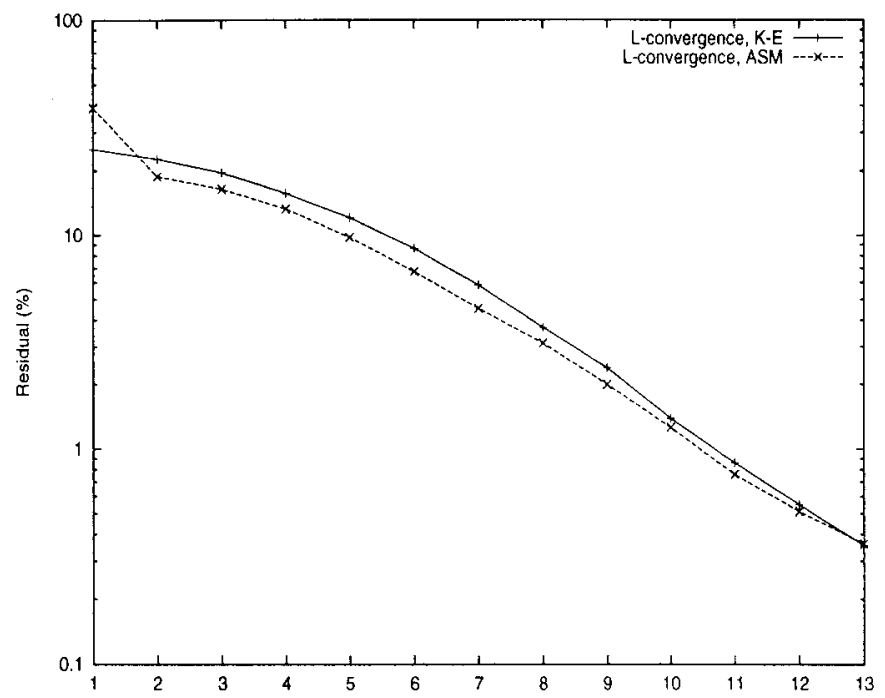

Figure 10. Convergence of $L$ for the flow in a polar cavity. First iteration of $\boldsymbol{u}$.

In order to observe the effect of $\Delta$, in Figure 16 a detail of the vortex in the symmetry plane is shown, first computing it with $\Delta=2 \mathrm{~mm}$ and then with $\Delta=7 \mathrm{~mm}$. Let $U_{*}$ be the solution of (17). In the case $\Delta=2 \mathrm{~mm}$, the parameter $y^{+}:=\Delta U_{*} / v$ verifies $30<y^{+}<100$ for all the boundary nodes, which is the condition of validity of the law of the wall. On the other hand, in the case $\Delta=7 \mathrm{~mm}$, there are nodes for which $y^{+}>100$. From Figure 16, it is clearly seen that the first case gives a solution closer to the experimental one than in the second case.

Concerning the convergence of the algorithm, a total of 45 outer iterations have been needed to converge to a tolerance of $0.1 \%$ in velocities (see Figure 17). From the 18th iteration, only one iteration for $L$ and one for $k$ and $\varepsilon$ is needed, except for iterations 26, 27 (three iterations) and 39 (two iterations).

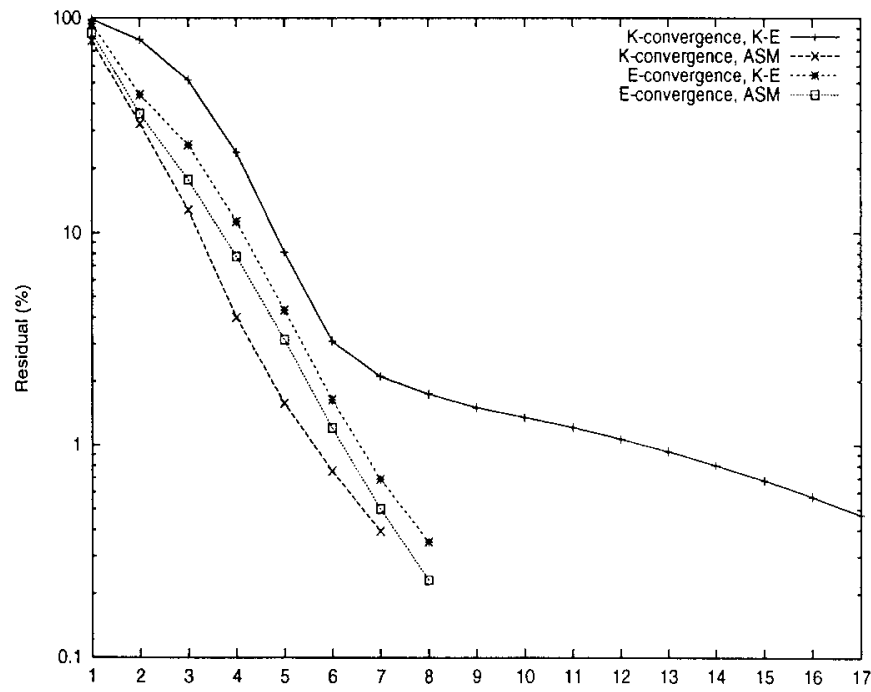

Figure 11. Convergence of $k$ and $\varepsilon$ for the flow in a polar cavity. First iteration of $\boldsymbol{u}$ and first iteration of $L$. 


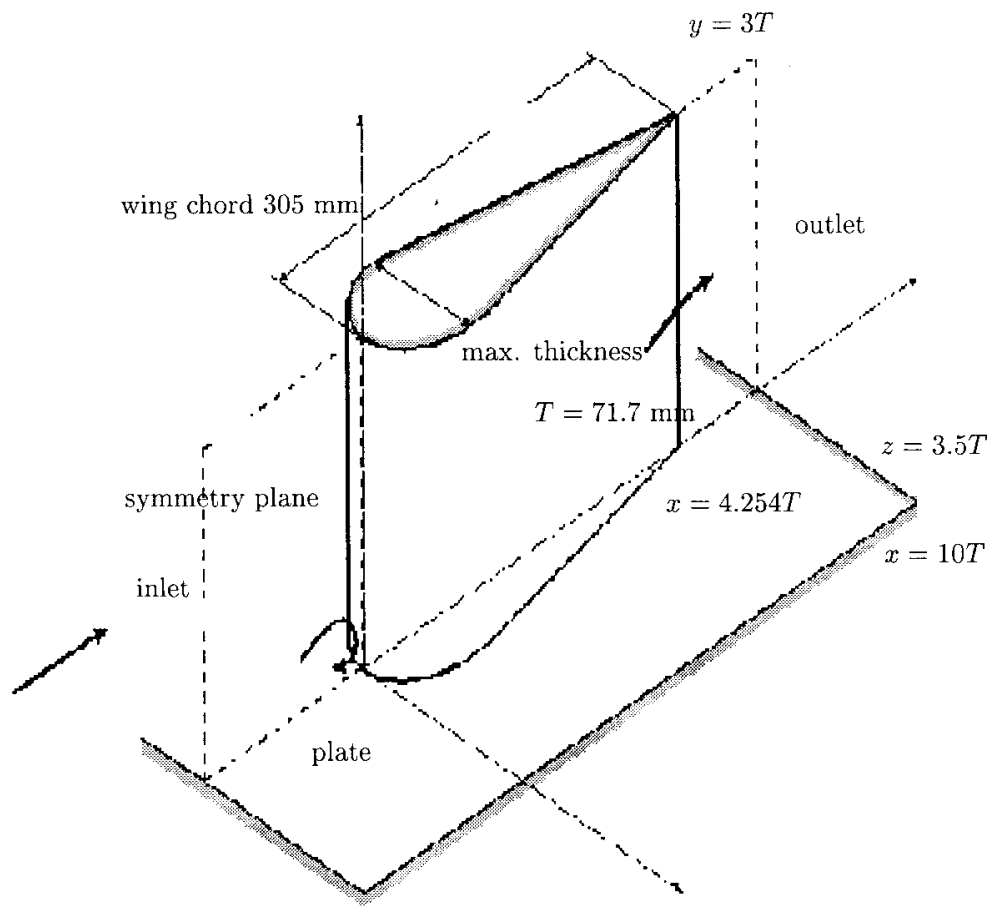

Figure 12. Flow over a wing: problem set-up.

\section{CONCLUSIONS}

In this paper, a numerical implementation of the standard $k-\varepsilon$ turbulence model for steady incompressible flows has been presented. The original features of the formulation presented herein are:

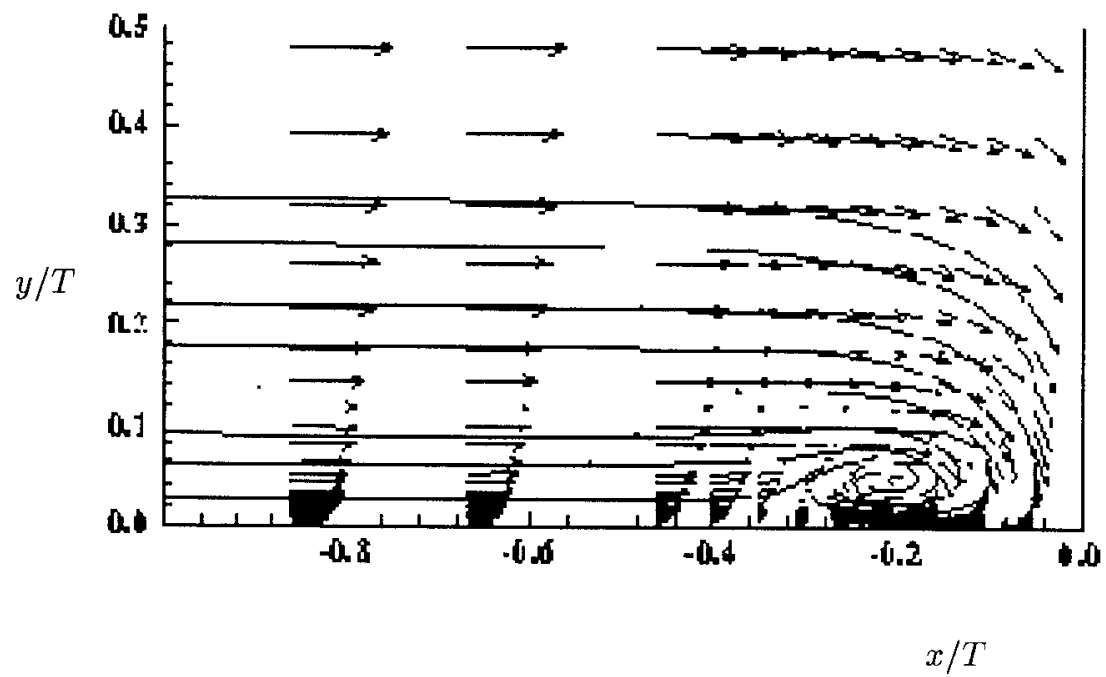

Figure 13. Experimental flow pattern at the symmetry plane for the flow over a wing (from Reference [27]). 


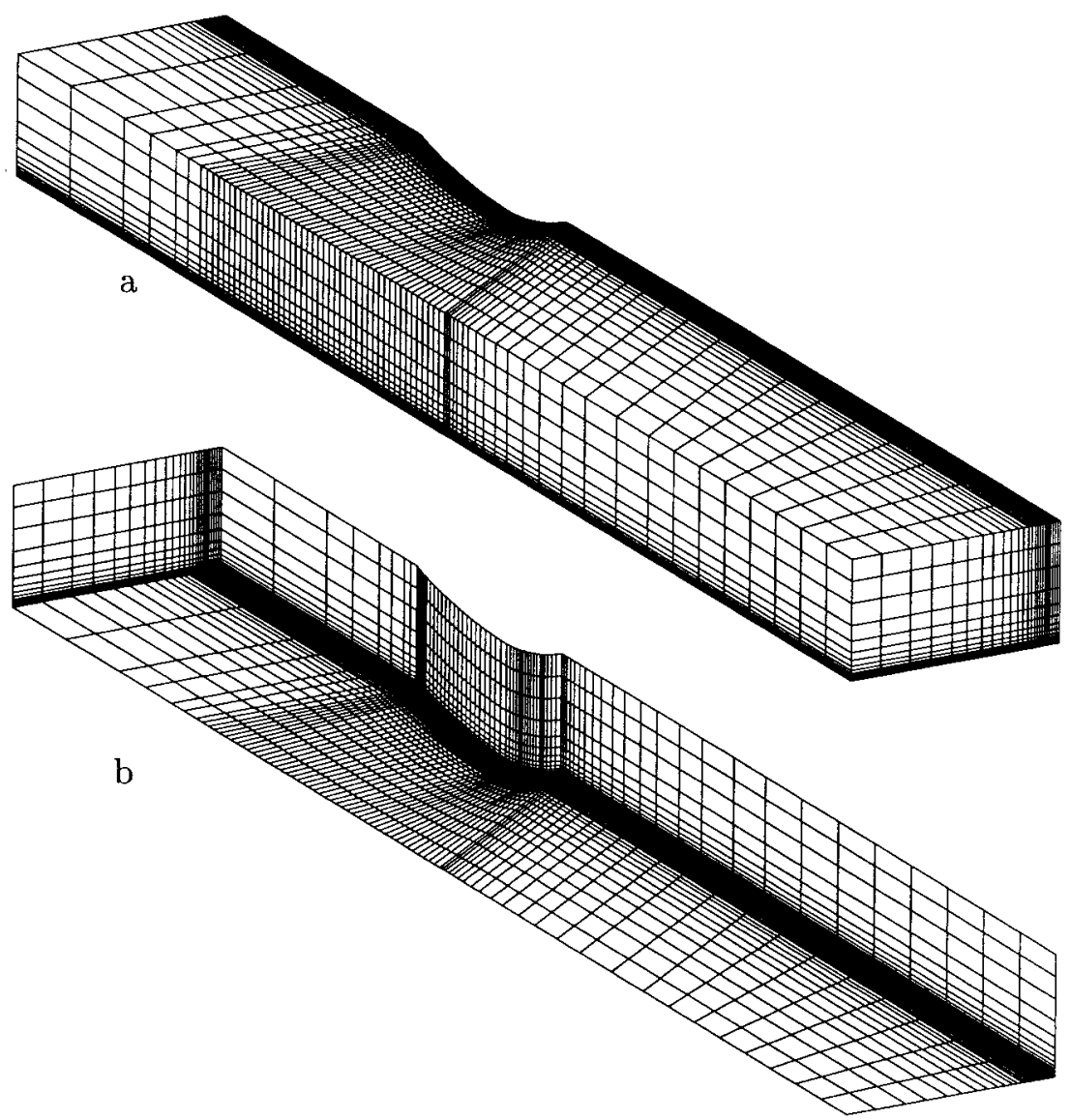

Figure 14. Surface mesh for the flow over a wing (37296 $Q_{1} / P_{0}$ elements, 41250 nodal points) (a) upper and frontal walls; (b) bottom and rear walls.

- A particular design of the linearization scheme based on three nested loops.

- A finite element approximation incorporating the use of a discontinuity capturing technique.

The authors have justified the use of both ingredients using heuristic arguments, and both have been found to be essential in order to have a robust scheme in general situations.

The leading idea for the design of the linearization has been the stability of the final linear equation to be solved, which is of convection-diffusion-reaction type. In the innermost loop, the diffusion coefficient and the reaction-like coefficient have been kept constant, in order to avoid the use of underestimated values of these coefficients. The authors have also applied this idea to the solution of the monolithic $k-\varepsilon$ system, i.e. solving the equations for $k$ and $\varepsilon$ directly coupled, although a better convergence of the iterative coupling described here has been obtained.

From numerical experiments it has been shown that the resulting numerical formulation is efficient, allowing to solve directly and accurately steady turbulent flows. To the authors knowledge, the use of discontinuity capturing techniques for turbulent incompressible flows has not been reported before. The numerical results indicate that accurate solutions may be 
obtained if this type of techniques (surely not only the particular one used in this paper) are properly used. It is well-known that one of the most important difficulties in the numerical approximation of turbulence models is the need for obtaining positive values of the turbulence parameters. The authors think that the use of discontinuity capturing methods may be of help to this end.

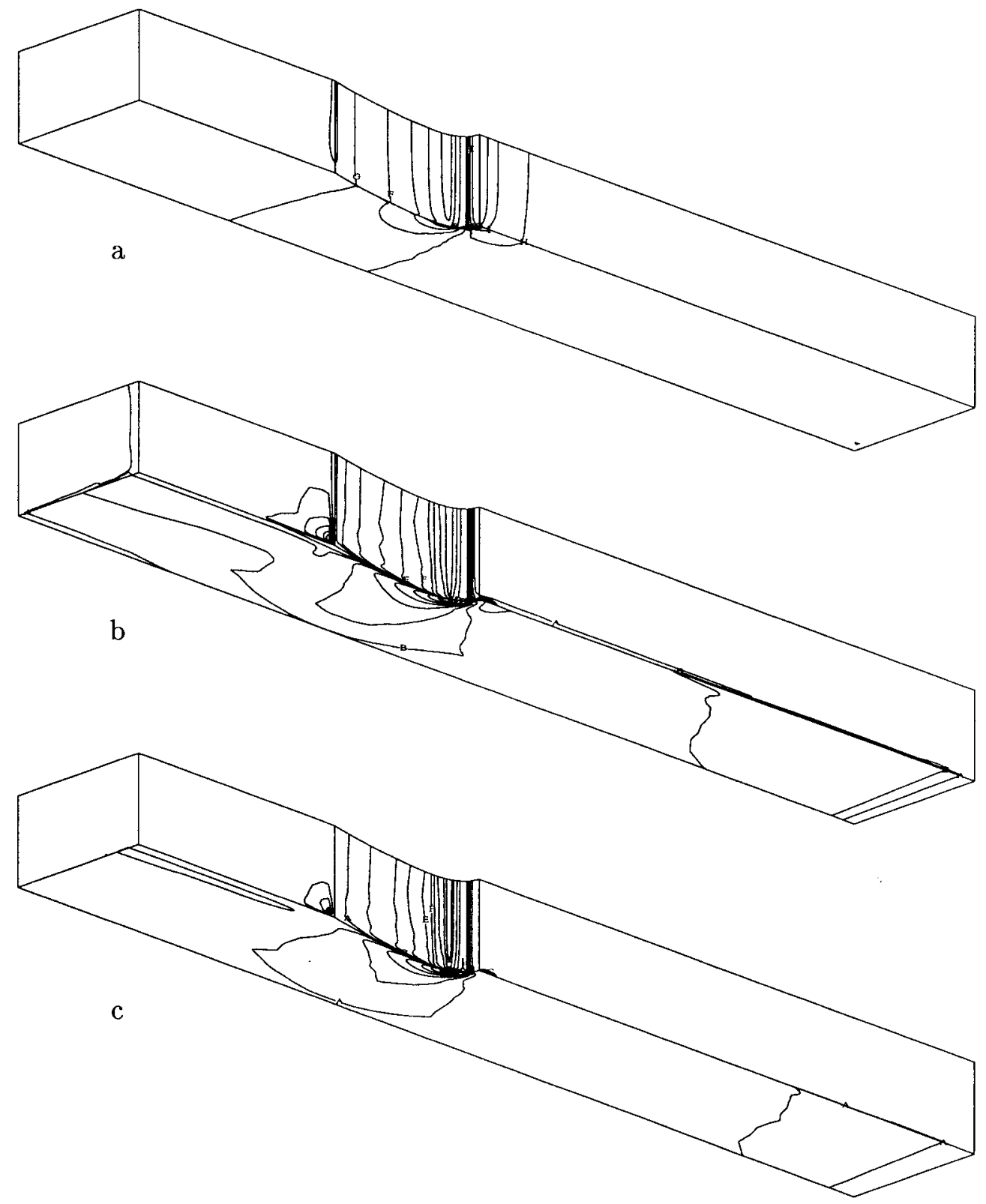

Figure 15. Results for the flow over a wing. (a) Pressure contours. (b) Contours of $k$. (c) Contours of $\varepsilon$. 


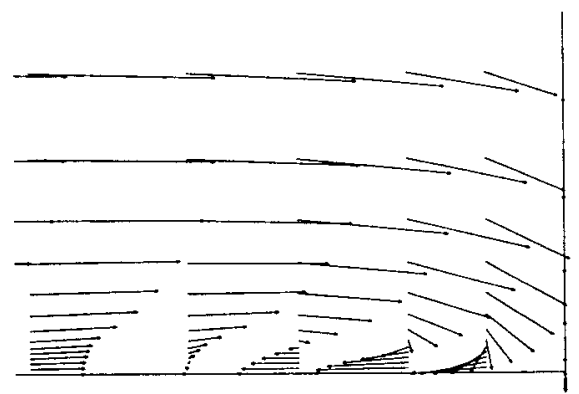

a

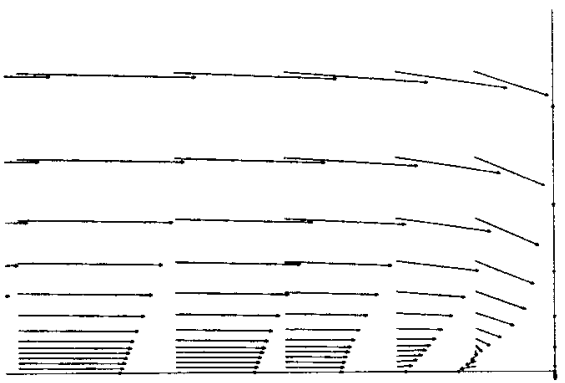

b

Figure 16. Detail of the vortex before the wing on the symmetry plane. (a) $\Delta=2 \mathrm{~mm}$. (b) $\Delta=7 \mathrm{~mm}$.

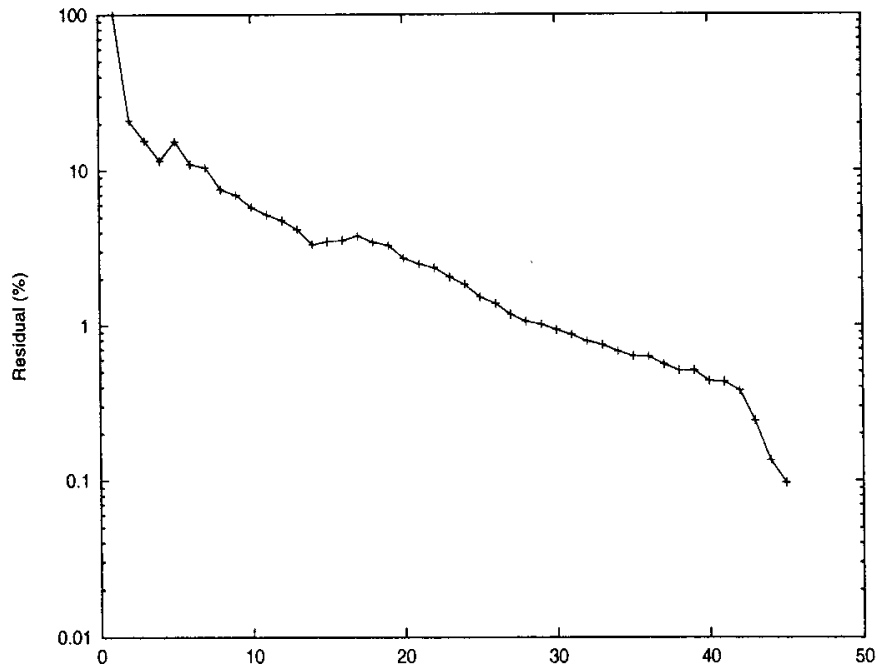

Figure 17. Velocity convergence for the flow over wing.

\section{APPENDIX A. NOMENCLATURE}

$c_{\mathrm{bc}}$

$c_{\mu}=0.09$

D

$e_{i j k}$

$f$

$f$

F

$\boldsymbol{g}_{1}, \boldsymbol{g}_{2}$

$\boldsymbol{G}$

$h^{e}$

$k$

$\boldsymbol{K}$

$L$ constant in the boundary condition for $k$

constant in turbulence modelling

discrete divergence matrix

components of the permutation tensor

source term of the model convection-diffusion-reaction (CDR) equation

vector of mean body forces

discrete force vector

unit tangent vectors to $\partial \Omega$

discrete gradient matrix

characteristic length of element $e\left(e=1, \ldots, n_{\mathrm{el}}\right)$

turbulent kinetic energy

matrix from viscous and convective terms of the Navier-Stokes equations mixing length 


$\begin{array}{ll}\boldsymbol{M}_{p} & \text { pressure mass matrix } \\ \boldsymbol{n} & \text { unit exterior normal to } \partial \boldsymbol{\Omega} \\ p & \text { mean pressure } \\ P e & \text { cell Péclet number } \\ P_{k} & \text { production term in the equation for } k \\ P_{\varepsilon} & \text { production term in the equation for } \varepsilon \\ \boldsymbol{P} & \text { vector of nodal pressures } \\ \mathscr{R}\left(\phi_{h}\right) & \text { element residual of the model CDR equation } \\ \boldsymbol{S}(\boldsymbol{u}) & \text { symmetric part of the mean velocity gradient } \\ \boldsymbol{t} & \text { traction vector } \\ \boldsymbol{u} & \text { mean velocity } \\ \boldsymbol{u}^{\prime} & \text { fluctuating velocity } \\ \boldsymbol{U} & \text { vector of nodal velocities } \\ \boldsymbol{W}(\boldsymbol{u}) & \text { skew-symmetric part of the mean velocity gradient }\end{array}$

Greek letters

$\begin{array}{ll}\alpha & \text { reaction coefficient of the model CDR equation } \\ \gamma & \text { relaxation parameter } \\ \partial \Omega & \text { boundary of } \Omega \\ \epsilon & \text { penalty parameter } \\ \varepsilon & \text { rate of turbulent energy dissipation } \\ \kappa & \text { Von Kármán constant/diffusivity of the CDR equation } \\ \kappa_{\mathrm{dc}}, \kappa_{\mathrm{s}}, \kappa_{\mathrm{SUPG}} & \text { various numerical diffusion parameters of the finite element approxima- } \\ v & \text { tion } \\ v_{t} & \text { kinematic viscosity } \\ \xi & \text { turbulent kinematic viscosity } \\ \rho & \text { upwind function of the SUPG method } \\ \sigma & \text { fluid density } \\ \tau & \text { Cauchy stress tensor } \\ \tau & \text { integral time scale/intrinsic time of the SUPG method } \\ \tau_{\mathrm{d}} & \text { Reynolds stress tensor } \\ \phi & \text { deviatoric part of the Reynolds stress tensor } \\ \phi_{h} & \text { scalar unknown of the model CDR equation } \\ \psi_{h} & \text { finite element approximation to } \phi \\ \omega & \text { finite element test function } \\ \omega & \text { dissipation per unit of kinetic energy } \\ \Delta & \text { speed of rotation vector } \\ \Phi_{h} & \text { distance from the wall at which the velocity is evaluated } \\ \Psi_{h} & \text { finite element space for } \phi_{h} \\ \Omega & \text { finite element space for } \psi_{h} \\ \Omega^{e} & \text { computational domain } \\ & \text { domain of element } e\left(e=1, \ldots, n_{\mathrm{el}}\right)\end{array}$

\section{REFERENCES}

1. J.O. Hinze, Turbulence, McGraw-Hill, New York, 1975.

2. B. Mohammadi and O. Pironneau, Analysis of the k-epsilon Turbulence Model, Wiley/Masson, New York, 1994.

3. W. Rodi, Turbulence Models and their Application in Hydraulics. A State of the Art Review, International Association for Hydraulic Research, Delft, 1980. 
4. R. Codina, 'A discontinuity-capturing cross-wind-dissipation for the finite element solution of the convectiondiffusion equation', Comput. Methods Appl. Mech. Eng., 110, 325-342 (1993).

5. R. Codina, 'A shock-capturing anisotropic diffusion for the finite element solution of the diffusion-convectionreaction equation', in Proc. VIII Int. Conf. on Finite Elements in Fluids, Part I, Barcelona, Spain, September 1993, CIMNE/Pineridge Press, 1993, pp. 67-75.

6. M.B. Goldschmit and M.A. Cavaliere, 'An iterative $(k-l)$ predictor $/ \varepsilon$ corrector algorithm for solving $(k-\varepsilon)$ turbulence models', Eng. Comput., 14, 441-455 (1997).

7. M. Jaeger and G. Dhatt, 'An extended $k-\varepsilon$ model', Int. J. Numer. Methods Fluids, 14, 1325-1345 (1992).

8. A. Autret, M. Grandotto and I. Dekeyser, 'Finite element computation of a turbulent flow over a two-dimensional backward-facing step', Int. J. Numer. Methods Fluids, 7, 89-102 (1987).

9. A.C. Benim and W. Zinser, 'Investigation into the finite element analysis of confined turbulent flows using a $k-\varepsilon$ model of turbulence', Comput. Methods Appl. Mech. Eng., 51, 507-523 (1985).

10. R.M. Smith, 'A practical method of two-equation turbulence modelling using finite elements', Int. J. Numer. Methods Fluids, 4, 321-336 (1984).

11. T. Utnes, 'Two-equation $(k-\varepsilon)$ turbulence computations by the use of a finite element model', Int. J. Numer. Methods Fluids, 8, 965-975 (1988).

12. A. Bakker and H.E.A. van den Akker, 'Single-phase flow in stirred reactors', Trans IChemE, 72, 583-593 (1994).

13. T.B. Gatski and C.G. Speziale, 'On explicit algebraic stress models for complex turbulent flows', J. Fluid Mech., 254, 59-78 (1993).

14. C.G. Speziale, 'Analytical methods for the development of Reynolds stress closures in turbulence', Annu. Rev. Fluid Mech., 23, 107-157 (1991).

15. M. Cervera, R. Codina and M. Galindo, 'On the computational efficiency and implementation of block-iterative algorithms for non-linear coupled problems', Eng. Comput., 13(6), 4-30 (1996).

16. D.C. Wilcox, Turbulence Modeling for CFD, DCW Industries, La Canada, CA, 1993.

17. D.C. Wilcox, 'Reassessment of the scale determining equation for advanced turbulence models', $A I A A J$., 26, $1299-1310$ (1988).

18. S. Zeierman and M. Wolfshtein, 'Turbulent time scale for turbulent-flow calculations', AIAA J., 24, 1606-1610 (1986).

19. A.N. Brooks and T.J.R. Hughes, 'Streamline upwind/Petrov-Galerkin formulations for convection dominated flows with particular emphasis on the incompressible Navier-Stokes equation', Comput. Methods Appl. Mech. Eng., 32, 199-259 (1982).

20. R. Codina, E. Oñate and M. Cervera, 'The intrinsic time for the streamline upwind/Petrov-Galerkin formulation using quadratic elements', Comput. Methods Appl. Mech. Eng., 94, 239-262 (1992).

21. A.C. Galeão and E.G. Dutra do Carmo, 'A consistent approximate upwind Petrov-Galerkin method for convection-dominated problems', Comput. Methods Appl. Mech. Eng., 68, 83-95 (1988).

22. C. Johnsom and A. Szepessy, 'On the convergence of a finite element method for a non-linear hyperbolic conservation law', Math. Comput., 49, 427-444 (1987).

23. F. Shakib, 'Finite element analysis of the compressible Euler and Navier-Stokes equations', Ph.D. Thesis, Stanford University, 1988.

24. F. Brezzi and M. Fortin, Mixed and Hybrid Finite Element Methods, Springer, Berlin, 1991.

25. R. Codina, 'An iterative penalty method for the finite element solution of the stationary Navier-Stokes equations', Comput. Methods Appl. Mech. Eng., 110, 237-262 (1993).

26. J. Kim, S.J. Kline and J.P. Johnston, 'Investigation of a reattaching turbulent shear layer: flow over a backward-facing step', J. Fluid Eng. ASME Trans., 102, 302-308 (1980).

27. ERCOFTAC, Database. http://fluindigo.mech.surrey.ac.uk/, University of Surrey, UK, 1996.

28. J.L. Fleming, R.L. Simpson, J.E. Cowling and W.J. Devenport, 'An experimental study of a turbulent wingbody junction and wake flow', Exp. Fluids, 14, 336 (1993).

29. J.Ch. Bonin, T. Buchal and W. Rodi, 'Data bases and testing of calculation methods for turbulent flows', ERCOFTAC Bull., 28, $48-54$ (1996). 